\title{
BUILDING SHADOW DETECTION FROM GHOST IMAGERY
}

\author{
Guoqing Zhou ${ }^{1}$, Junhong Sha ${ }^{1}$, Tao Yue ${ }^{1,}{ }^{*}$, Qingyang Wang ${ }^{1}$, Xiaofan Liu ${ }^{1}$, Shengxin Huang ${ }^{1}$, Qiuyu Pan ${ }^{1}$, Jiandong Wei ${ }^{1,2}$ \\ ${ }^{1}$ Guangxi Key Laboratory of Spatial Information and Geomatics, Guilin University of Technology, No. 12 Jian'gan Road, Guilin, \\ Guangxi 541004, China - (gzhou, yuetao)@glut.edu.cn \\ ${ }^{2}$ Department of Mechanical and Control Engineering, Guilin University of Technology, No. 12 Jian'gan Road, Guilin, Guangxi \\ 541004, China-961466473@qq.com
}

\section{Commission Urban Sensing and Mobility}

KEY WORDS: Ghost image, Interior and exterior orientation elements, Zenith angle of sun, Region growing method, Urban large-scale aerial imagines

\begin{abstract}
:
Shadow is one of the basic features of remote sensing image, it expresses a lot of information of the object which is loss or interference, and the removal of shadow is always a difficult problem to remote sensing image processing. In this paper, it is mainly analyzes the characteristics and properties of shadows from the ghost image (traditional orthorectification). The DBM and the interior and exterior orientation elements of the image are used to calculate the zenith angle of sun. Then this paper combines the scope of the architectural shadows which has be determined by the zenith angle of sun with the region growing method to make the detection of architectural shadow areas. This method lays a solid foundation for the shadow of the repair from the ghost image later. It will greatly improve the accuracy of shadow detection from buildings and make it more conducive to solve the problem of urban large-scale aerial imagines.
\end{abstract}

\section{INTRODUCTION}

With the rapid development of space technology and remote sensing technology, The application of high resolution aerial image is more and more extensive. In the process of aerial image imaging, tall buildings, trees and other objects have a serious impact on the quality of the image (Zhou, 2004, 2005a). Therefore, in the image, there are shadows of tall buildings, trees, etc., which restrict the effective use of aerial images.The shadow not only has serious influence on the automatic detection of the objects, image recognition and classification, image pattern matching, but also affects the integrity of image digital information.In dynamic real-time monitoring, the shadow is more likely to make the target image and target fusion error. Early in the middle and late twentieth Century a large number of domestic and foreign scholars began to work on processing the shadows of aerial images. These methods can be divided into two categories: one is based on the model and the other is based on the nature of the shadow. In the first class, the model refers to the three-dimensional geometry of the scene and the known information of the light source, then calculating the shadow region (Zhou, 2005). However, the 3D structure of the light source and scene is not easy to obtain, thus the limitations of these methods are large relatively.The second type is more universal, identifying shadows by analyzing the properties of shadows in terms of width, geometry and color.The second type of methods has a wide range of application.However, this kind of shadow detection method is still insufficient, which is a method of different features about the colour and the ability to adapt to the image of resolution is poor. The method which is based on texture feature need to use the pixel digital number around it to calculate the result, the influence factors of shadow are numerous, different regions are different, some parameters are difficult. the selection of optimal threshold will affect the shadow detection; The method which is based on the edge feature, it is not only the edges of the shadow are needed, but also the feature points and coordinates of the architectural edges may be needed. The real shadow edges need to be distinguished from all the edges (Liu, 2012; Li, 2004, 2005b; Valo, 2008). In this paper the method of shadow detection is the first method.The method is based on digital buildings model, and the model provided by the structure of the three dimensional space information (Barazzetti, 2010; Deng, 2015). These interior and exterior orientation elements of original image are combined to calculate the zenith angle of sun, then the scope of the shadows from the buildings will be gained.The regional growth method is used to determine the shadow region accurately. The experimental results show that this method can greatly improve the efficiency and precision of shadow detection (Rau, 2002). The method also makes it more conducive to solve the problem of urban large-scale aerial imagines.

\section{GHOST IMAGERY}

Because the traditional orthorectification is the process of correcting the local presence of point displacement, in the process of orthorectification. The image points are resampled and distributed according to the location of each grid from the DSM. The process of gray-scale resampling is determined based on the grayscale value which is near the image point from this image. But, in fact, the architectural wall which is vertical to the ground on the orthophoto there is no image when the process of ortho-rectificationis carried out to the part of the building walls, that is to say, in the process of orthorectification, the image of the building is just has its roof (through orthorectification, the roof of the building will be correctted to the image in the correct position according to architectural geographic space position from its DSM, and then processing it by the gray resampling method, forming the correct location of

\footnotetext{
* Corresponding author:Tao Yue, Email:yuetao@glut.edu.cn
} 
building roof image in the orthoimage). In the original image, because of the influence of perspective projection, the wall of the building will also be imaged in the image, and this part of the wall will not be detected by penetrating the correct process, therefore, the wall of the building has shade on the ground or low-rise building also leds to the building has its ghosting phenomenon in the orthoimage, this phenomenon is called the ghost image (Zhou, 2017). As shown in the Figure 1.

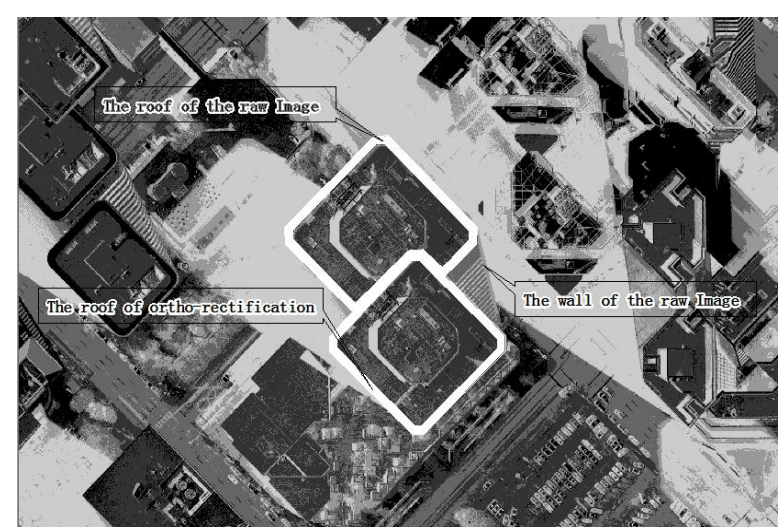

Figure 1. Ghost image

\section{SHADOW DETECTION}

The shadows of the buildings are mainly the shading of the tall buildings on the ground from the sunlight, and the darker areas are produced in the corresponding position. The shadow can be regarded as a special kind of noise, or it can be understood as a special form of image degradation, which is the distortion of the building target information in the shadow area.The shadow is divided into umbra and self-shadow. Since the part of the architectural wall is not visible in the positive projective image, this paper mainly carries out experimental analysis on the umbra of the building (Zhou, 2016). The paper mainly calculates the zenith angle of sun through the DBM and the interior and exterior orientation elements. In order to determine the shadow range of the buildings, the coordinates of the shaded boundary needed to be calculated. In the end, the region growing method is used to determine the shadow region accurately.

\subsection{The Calculation of the Zenith Angle of Sun}

The sunlight is parallel, so the zenith angle of sun to each building target in the same image is the same constant value. According to the interior and exterior orientation elements of the original image and the digital building model of it. We can get the earth coordinate of one point $A$ is:

$$
\begin{aligned}
& X_{A}=\left(Z_{A}-Z_{S}\right) \frac{a_{1} x+a_{2} y-a_{3} f}{c_{1} x+c_{2} y-c_{3} f}+X_{s} \\
& Y_{A}=\left(Z_{A}-Z_{S}\right) \frac{b_{1} x+b_{2} y-b_{3} f}{c_{1} x+c_{2} y-c_{3} f}+Y_{S} \\
& Z_{A}=Z_{0}
\end{aligned}
$$

Using the digital building model the length of ${ }^{L}$ and the height of the building can be calculated:

$$
\begin{gathered}
L=\sqrt{\left(X_{D^{\prime}}-X_{A}\right)^{2}+\left(Y_{D^{\prime}}-Y_{A}\right)^{2}} \\
H=Z_{D}-Z_{D^{\prime}}
\end{gathered}
$$

A wall of the building corresponds to a boundary in the shaded area, since the wall is perpendicular to the ground.The principle is shown in the Figure 2. Therefore, the Zenith angle of sun $\alpha$ is calculated as follow:

$$
\alpha=\arctan \frac{D D^{\prime}}{D^{\prime} A}=\frac{H}{L}
$$

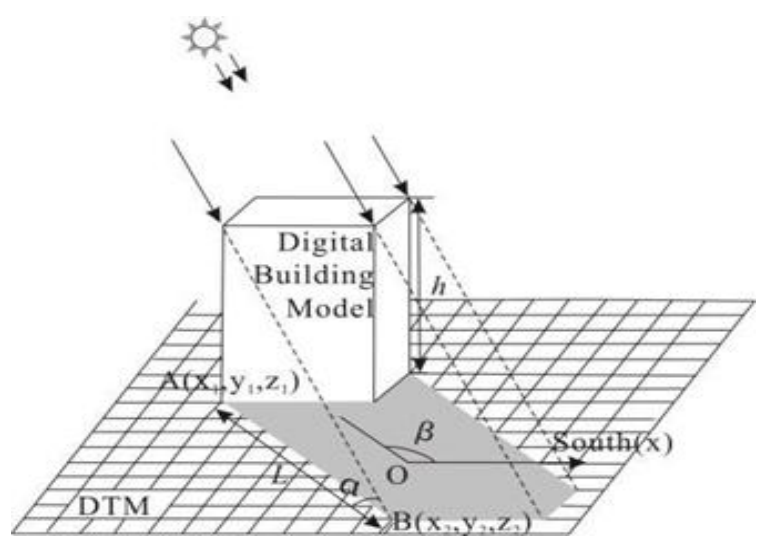

Figure 2 . The zenith angle of the sun $\beta$ and the altitude angle of the sun $\alpha$; h is the height of the building; $L$ is the length of the shadow (Zhou, 2015)

\subsection{The calculation of shadow boundary coordinates}

According to the theory that the sun's rays are parallel, the rays of the sun's rays are parallel to the ground. And assuming the direction vector is $(0,1)$, That's the Angle between the positive and the north is $\beta$ :

$$
\beta=\arccos \frac{\mathrm{Y}_{\mathrm{A}}-\mathrm{Y}_{\mathrm{D}^{\prime}}}{\sqrt{\left(\mathrm{Y}_{\mathrm{A}}-\mathrm{Y}_{\mathrm{D}^{\prime}}\right)^{2}+\left(\mathrm{X}_{\mathrm{A}}-\mathrm{X}_{\mathrm{D}^{\prime}}\right)^{2}}}
$$

After determining the $\mathrm{C}$ point coordinates by $\alpha, \beta$, the collinear equation is used to solve the position coordinate of the image point $\alpha$ :

$$
\begin{aligned}
& x a=-f \frac{a 1\left(X_{C}-X_{S}\right)+b 1\left(Y_{C}-Y_{S}\right)+c 1\left(Z_{0}-Z_{s}\right)}{a 3\left(X_{C}-X_{S}\right)+b 3\left(Y_{C}-Y_{S}\right)+c 3\left(Z_{0}-Z_{s}\right)} \\
& y a=-f \frac{a 2\left(X_{C}-X_{S}\right)+b 2\left(Y_{C}-Y_{S}\right)+c 2\left(Z_{0}-Z_{s}\right)}{a 3\left(X_{C}-X_{S}\right)+b 3\left(Y_{C}-Y_{S}\right)+c 3\left(Z_{0}-Z s\right)}
\end{aligned}
$$

Then the boundary coordinates of all the buildings in the range are calculated to determine the shaded region. 


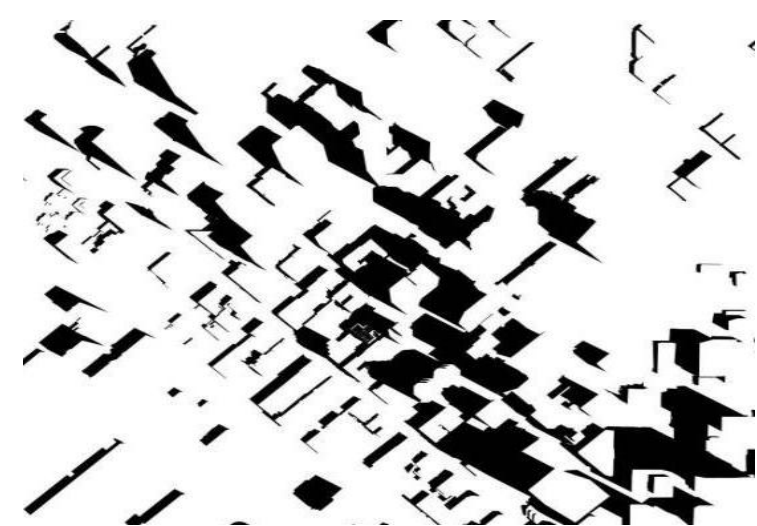

Figure 3 . The shaded regionof all the buildings in the range

\subsection{Region Growing Method}

The basic idea of regional growing method is to assemble the pixels of the similarity from the region.Firstly, it needs to find a seed pixel as the growth of seven points, and then the neighborhood pixels have the same or similarity with the seed quality (according to the pre-determined growth or similarity criterion to determine) incorporated into the seed pixels in the area.The new pixel continues to grow as a seed, until the pixels
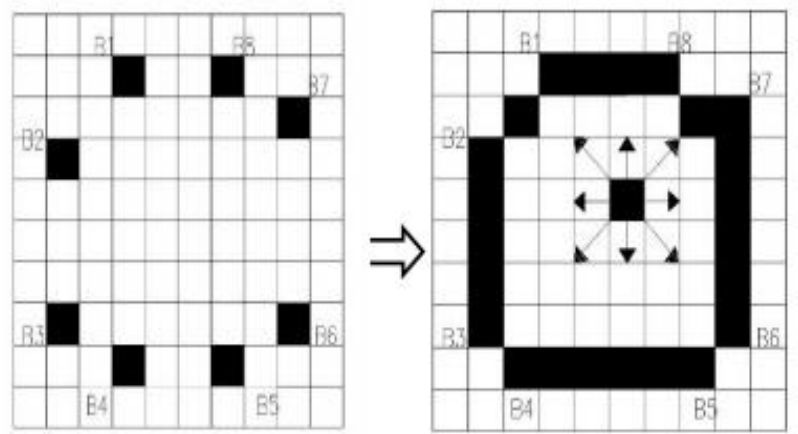

Figure 4. The region growth method gets the image points in the shadow region

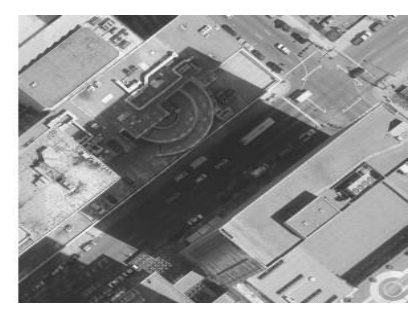
the original image
Figure 6. The shadowed of

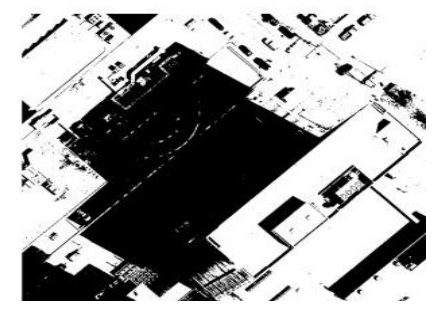

Figure 7. The shadow region of regional growth method
A large number of experimental results show: the detection result of the Figure 7 has the value of gray value in the shadow region of the shadow region. It can be seen that the method of the Figure 8 can effectively detect the shadow area of a single building. But the test results still contained non-shadow objects. Therefore, the detection accuracy of the method used in this paper is higher than other methods (Figure 9). From the above data, it can be seen that the method mentioned in this paper greatly improves the precision of shadow detection.
This experiment to extract the roofs of the ghost image, and then determine the shaded area boundary and 8 connected region growing method is used to obtain all shadow region within the image points and will get the grey value of image point is set to 0 . In the end , the extract the ghost image roof overlaid on the image.

\section{EXPERIMENT RESULTS AND ANALYSIS}

The section is the experiment and results of the shadow detection which contrasts the different methods to prove our method accurate. The experimental field is located in downtown Denver, Colorado. The original aerial image from one flight strips were acquired using an RC30 aeria. The aerial images (dv1119) covers the downtown area with approximately $65 \%$ endlap and $30 \%$ sidelap. The final experimental result is shown in the Figure 5.

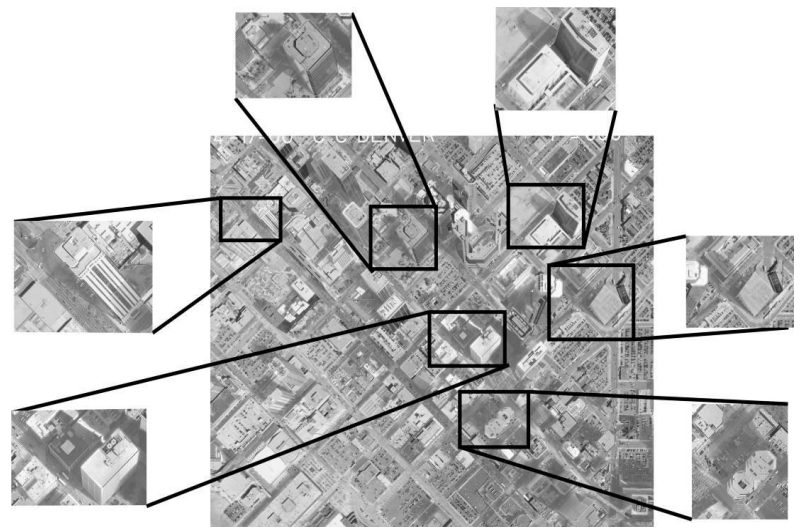

Figure 5. The final experimental result

Comparing several methods, the experimental results of this paper are as follows. The Figure 6 is a building in the original image and its shadowed area. The Figure 7 is the results of regional growth in the shadow region of the building. The Figure 8 calculate the boundary coordinates of the shadow region of the building based on the DBM geometry method. The Figure 9 is the purposes of this article.

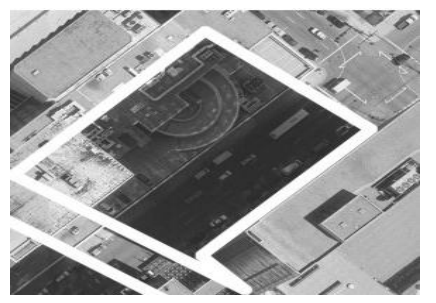

Figure 8 . The shadow based on the DBM geometry method

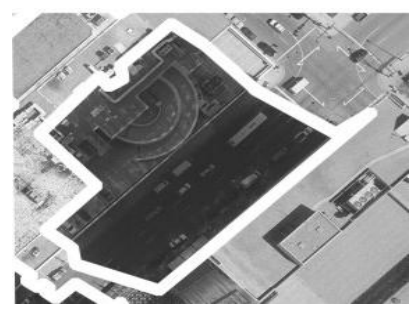

Figure 9. The result of the method used in this article

\section{CONCLUSION}

By comparing the original image of shadow zone with the shadow region of regional growth method and the shadow based on the DBM geometry method, we can know that our method will greatly improve the accuracy of building shadow detection. The result of this study would be useful for the automatic detection of the objects, image recognition and classification, image pattern matching, as well as the result of this study also can improve the integrity of the digital 
information from the image and make it more conducive to solve the problem of urban large-scale aerial imagines.

\section{ACKNOWLEDGEMENTS}

This paper is supported by the National Natural Science of China under Grant numbers 41431179, the National Key Research and Development Program of China under Grant numbers 2016YFB0502500, the State Oceanic Administration under Grant numbers 2014\#58, GuangXi Natural Science Foundation under grant numbers 2015GXNSFDA139032, Guangxi Science \& Technology Development Program under the Contract numbers GuiKeHe 14123001-4, GuangXi Key Laboratory of Spatial Information and Geomatics Program under Grant numbers 151400701, 151400712, 163802512, Guangxi Doctoral student innovation under grant numbers YCBZ2018054.

\section{REFERENCES}

Barazzetti L., Brovelli M. and Valentini L., 2010. LiDAR digital building models for true orthophoto generation. Applied Geomatics. 2(4), pp.187-196.

Deng F., Kang J., Li P. and Wan F., 2015. Automatic true orthophoto generation based on three-dimensional building model using multiview urban aerial images. Journal of Applied Remote Sensing. 9(1),095087.

Liu W. and Yamazaki F., 2012. Object-Based Shadow Extraction and Correction of High-Resolution Optical Satellite Images. IEEE Journal of Selected Topics in Applied Earth Observations \& Remote Sensing. 5(4), pp. 1296-1302.

Li Y., Gong P. and Sasagawa T., 2005. Integrated shadow removal based on photogrammetry and image analysis International Journal of Remote Sensing. 26(18), pp. 3911-3929.

Massalabi A., He D.C., Bénié, G.B. and Beaudry E., 2004. Restitution of information under shadow in remote sensing high space resolution images: application to IKONOS data of Sherbrooke city. International Archives of the Photogrammetry, Remote Sensing and Spatial Information Sciences, 35 (Part B3), pp. 173-178.

Chen N., Chen L. and Rau J., 2002. True orthophotogeneration of built-up areas using multi-view images. Photogrammetric Engineering and Remote Sensing. 68(6), pp. 581-588.

Ambrosio G., 2008. Shadow detection in colour high-resolution satellite images. Taylor \& Francis, Inc.

Zhou G., Song C. and Schickler W., 2004. Urban 3D GIS from LIDAR and aerial image data. Computers and Geosciences. 30(4), pp. 345-353.

Zhou G., Han C., Ye S., Wang Y., and Wang C., 2015. An integrated approach for shadow detection of the building in urban areas// International Conference on Intelligent Earth Observing and Applications. International Society for Optics and Photonics, 98082W.

Zhou G. and Song P., 2005a. Urban 3D mapping from airborne image flow data. International Journal of Remote Sensing. 26 (1), pp. 79-99.
Zhou G., Chen W. and Kelmelis J., 2005b. A comprehensive study on urban aerial image orthorectification for national mapping program. IEEE Trans. on Geosciene and Remote Sensing. 43(9), pp. 2138-2147.

Zhou G., Wang Y., Yue T. and Ye S., 2017. Building Occlusion detection from ghost imagery. IEEE Trans. on Geoscience and Remote Sensing. 55(2), pp. 1074 -1084.

Zhou G., 2016. Study on Solar Tracking System based on Image Shadow Detection Technology. Journal of residuals Science\& Technology. 32(4), pp. 245-249. 